\title{
FUZZY VOLTERRA INTEGRAL EQUATIONS WITH INFINITE DELAY
}

\author{
P. PRAKASH AND V. KALAISELVI
}

\begin{abstract}
In this paper, we study the existence and uniqueness of solutions for a class of fuzzy Volterra integral equations with infinite delay by using the method of successive approximations.
\end{abstract}

\section{Introduction}

Kandel and Byatt [7] introduced the concept of fuzzy differential equations. Later it was applied in fuzzy processes and fuzzy dynamical systems. Since 1987, the Cauchy problem for first order fuzzy differential equations has been extensively investigated by Kaleva [6]. Song et al. [10] discussed about the existence and comparison theorems to Volterra fuzzy integral equations in $\left(E^{n}, D\right)$. Diamond [4] discussed the theory of Volterra integral equations in a fuzzy context by using the interpretation equations. Balachandran and Prakash [3] studied the existence of solutions of nonlinear fuzzy Volterra-Fredholm integral equations by using the Successive approximation method. Agarwal et al. [1] have given a very general formulation of the stacking theorem approach for fuzzy Volterra integral equations. Recently, Xiaoping and Yongiang [11] establish the existence and uniqueness of solutions for set differential equations, the continuous dependence of solutions on initial values, and the structural stability of solutions by using the Banach fixed point theorem. They also discussed the relationship between small solutions and large solutions of fuzzy differential equations.

In this paper, we consider the fuzzy Volterra integral equation with infinite delay of the form

$$
x^{\prime}(t)=h(t, x(t))+\int_{-\infty}^{t} q(t, s, x(s)) d s, \quad t \in T=(-\infty, \infty)
$$

where $h: T \times E^{n} \rightarrow E^{n}$ and $q: T \times T \times E^{n} \rightarrow E^{n}$ are levelwise continuous and satisfy the generalized Lipschitz conditions.

Basic Assumption: For each $t_{0} \in T$, there exists a nonempty convex subset $B\left(t_{0}\right)$ of the space of continuous functions $\phi: T_{1}=\left(-\infty, t_{0}\right] \rightarrow E^{n}$ such that $\phi \in B\left(t_{0}\right)$ implies

$$
\int_{-\infty}^{t_{0}} q(t, s, \phi(s)) d s:=Q\left(t, t_{0}, \phi\right)
$$

Received January 15, 2008.

2000 Mathematics Subject Classification. 45G10, 45M10.

Key words and phrases. Fuzzy set-valued mapping, levelwise continuous, fuzzy Volterra integral equations, fuzzy solution. 
is continuous on $T_{2}=\left[t_{0}, \infty\right)$. For a given $t_{0} \in T$ and a continuous initial function $\phi: T_{1} \rightarrow E^{n}$, we seek a continuous solution $x\left(t, t_{0}, \phi\right)$ satisfying (1) for $t \in\left[t_{0}, t_{0}+\beta\right)$ for some $\beta>0$ with $x\left(t, t_{0}, \phi\right)=\phi(t)$ for $t \leq t_{0}$.

\section{Preliminaries}

Let $P_{K}\left(R^{n}\right)$ denote the family of all nonempty, compact, convex subsets of $R^{n}$. Addition and scalar multiplication in $P_{K}\left(R^{n}\right)$ are defined as usual. Let $A$ and $B$ be two nonempty bounded subsets of $R^{n}$. The distance between $A$ and $B$ is defined by the Hausdorff metric

$$
d(A, B)=\max \left\{\sup _{a \in A} \inf _{b \in B}\|a-b\|, \sup _{b \in B} \inf _{a \in A}\|a-b\|\right\},
$$

where $\|\cdot\|$ denotes the usual Euclidean norm in $R^{n}$. Then it is clear that $\left(P_{K}\left(R^{n}\right), d\right)$ becomes a complete metric space [9].

Let $I=[0,1] \subseteq R$ be a compact interval and let $E^{n}$ denote the set of all $u: R^{n} \rightarrow I$ such that $u$ satisfies the following conditions.

(i) $u$ is normal, that is, there exists an $x_{0} \in R^{n}$ such that $u\left(x_{0}\right)=1$,

(ii) $u$ is fuzzy convex,

(iii) $u$ is upper semicontinuous,

(iv) $[u]^{0}=\operatorname{cl}\left\{x \in R^{n}: u(x)>0\right\}$ is compact.

For $0<\alpha \leq 1$, denote $[u]^{\alpha}=\left\{x \in R^{n}: u(x) \geq \alpha\right\}$. Then from (i)-(iv) it follows that the $\alpha$-level set $[u]^{\alpha} \in P_{K}\left(R^{n}\right)$ for all $0 \leq \alpha \leq 1$.

If $g: R^{n} \times R^{n} \rightarrow R^{n}$ is a function, then using Zadeh's extension principle,we can extend $g$ to $E^{n} \times E^{n} \rightarrow E^{n}$ by the equation

$$
\tilde{g}(u, v)(z)=\sup _{z=g(x, y)} \min \{u(x), v(y)\} .
$$

It is well known that $[\tilde{g}(u, v)]^{\alpha}=g\left([u]^{\alpha},[v]^{\alpha}\right)$ for all $u, v \in E^{n}, 0 \leq \alpha \leq 1$ and any continuous function $g$. Furthermore, we have $[u+v]^{\alpha}=[u]^{\alpha}+[v]^{\alpha}$ and $[k u]^{\alpha}=k[u]^{\alpha}$, where $k \in R$.

Theorem 2.1.([8]) If $u \in E^{n}$, then

(i) $[u]^{\alpha} \in P_{K}\left(R^{n}\right)$ for $0 \leq \alpha \leq 1$,

(ii) $[u]^{\alpha_{2}} \subset[u]^{\alpha_{1}}$ for $0 \leq \alpha_{1} \leq \alpha_{2} \leq 1$, and

(iii) If $\left\{\alpha_{k}\right\} \subset[0,1]$ is a nondecreasing sequence converging to $\alpha>0$, then

$$
[u]^{\alpha}=\bigcap_{k \geq 1}[u]^{\alpha_{k}} .
$$


Conversely, if $\left\{A^{\alpha}: 0 \leq \alpha \leq 1\right\}$ is a family of subset A of $R^{n}$ satisfying (i)-(iii), then there exists a $u \in E^{n}$ such that

$$
[u]^{\alpha}=A^{\alpha} \text { for } 0<\alpha \leq 1
$$

and

$$
[u]^{0}=\overline{\bigcup_{0<\alpha \leq 1} A^{\alpha}} \subset A^{0} .
$$

Define the metric $D: E^{n} \times E^{n} \rightarrow R^{+} \cup\{0\}$ by $D(u, v)=\sup _{0 \leq \alpha \leq 1} d\left([u]^{\alpha},[v]^{\alpha}\right)$, where $d$ is the Hausdorff metric defined in $P_{K}\left(R^{n}\right)$.

The following definitions are given in [5].

Definition 2.1. A mapping $F: I \rightarrow E^{n}$ is strongly measurable, if for all $\alpha \in[0,1]$ the setvalued mapping $F_{\alpha}: I \rightarrow P_{K}\left(R^{n}\right)$ defined by $F_{\alpha}(t)=[F(t)]^{\alpha}$ is Lebesgue measurable when $P_{K}\left(R^{n}\right)$ has the topology induced by the Hausdorff metric $d$.

Definition 2.2. A mapping $F: I \rightarrow E^{n}$ is called levelwise continuous at $t_{0} \in I$ if the setvalued mapping $F_{\alpha}(t)=[F(t)]^{\alpha}$ is continuous at $t=t_{0}$ with respect to the Hausdorff metric $d$ for all $\alpha \in[0,1]$.

Definition 2.3. A mapping $F: I \rightarrow E^{n}$ is called integrably bounded if there exists an integrable function $h$ such that $\|x\| \leq h(t)$ for every $x \in F_{0}(t)$.

Definition 2.4. The integral of a fuzzy mapping $F: I \rightarrow E^{n}$ is defined levelwise by $\left[\int_{I} F(t) d t\right]^{\alpha}=$ $\int_{I} F_{\alpha}(t) d t=$ The set of all $\int_{I} f(t) d t$ such that $f: I \rightarrow R^{n}$ is a measurable selection for $F_{\alpha}$ for all $\alpha \in[0,1]$.

Theorem 2.2. ([2]) If $F: I \rightarrow E^{n}$ is strongly measurable and integrably bounded, then $F$ is integrable.

It is known that $\left[\int_{I} F(t) d t\right]^{0}=\int_{I} F_{0}(t) d t$.

Theorem 2.3. Let $F, G: I \rightarrow E^{n}$ be integrable and $\lambda \in R$. Then

(i) $\int_{I}(F(t)+G(t)) d t=\int_{I} F(t) d t+\int_{I} G(t) d t$,

(ii) $\int_{I} \lambda F(t) d t=\lambda \int_{I} F(t) d t$,

(iii) $D(F, G)$ is integrable,

(iv) $D\left(\int_{I} F(t) d t, \int_{I} G(t) d t\right) \leq \int_{I} D(F(t), G(t)) d t$.

Definition 2.5. A mapping $F: I \rightarrow E^{n}$ is called differentiable at $t_{0} \in I$ if, for any $\alpha \in[0,1]$, the set-valued mapping $F_{\alpha}(t)=[F(t)]^{\alpha}$ is Hukuhara differentiable at $t_{0}$ with $D F_{\alpha}\left(t_{0}\right)$ and the family $\left\{D F_{\alpha}\left(t_{0}\right) \mid \alpha \in[0,1]\right\}$ define a fuzzy number $F\left(t_{0}\right) \in E^{n}$. 
If $F: I \rightarrow E^{n}$ is differentiable at $t_{0} \in I$, then we say that $F^{\prime}\left(t_{0}\right)$ is the fuzzy derivative of $F(t)$ at the point $t_{0}$.

Theorem 2.4. Let $F: I \rightarrow E^{1}$ be differentiable. Denote $F_{\alpha}(t)=\left[f_{\alpha}(t), g_{\alpha}(t)\right]$. Then $f_{\alpha}$ and $g_{\alpha}$ are differentiable and $\left[F^{\prime}(t)\right]^{\alpha}=\left[f_{\alpha}^{\prime}(t), g_{\alpha}^{\prime}(t)\right]$.

Theorem 2.5. Let $F: I \rightarrow E^{n}$ be differentiable and assume that the derivative $F^{\prime}$ is integrable over I. Then, for each $s \in I$, we have

$$
F(s)=F(a)+\int_{a}^{s} F^{\prime}(t) d t .
$$

Definition 2.6. A mapping $f: I \times E^{n} \rightarrow E^{n}$ is called levelwise continuous at point $\left(t_{0}, x_{0}\right) \in$ $I \times E^{n}$ provided, for any fixed $\alpha \in[0,1]$ and arbitrary $\epsilon>0$, there exists a $\delta(\epsilon, \alpha)>0$ such that

$$
d\left([f(t, x)]^{\alpha},\left[f\left(t_{0}, x_{0}\right)\right]^{\alpha}\right)<\epsilon
$$

whenever $\left|t-t_{0}\right|<\delta(\epsilon, \alpha)$ and $d\left([x]^{\alpha},\left[x_{0}\right]^{\alpha}\right)<\delta(\epsilon, \alpha)$ for all $t \in I, x \in E^{n}$.

\section{Main results}

Assume that $h: T_{0} \times E^{n} \rightarrow E^{n}$ and $q: T_{0} \times T_{0} \times E^{n} \rightarrow E^{n}$ are levelwise continuous, where $T_{0}=\left\{t \in T: t_{0} \leq t<t_{0}+\beta\right\}$. Consider the fuzzy Volterra integral equation (1) where $\phi\left(t_{0}\right) \in E^{n}$. We denote $J=T_{0} \times B\left(\phi\left(t_{0}\right), b\right)$ and $J_{0}=T_{0} \times T_{0} \times B\left(\phi\left(t_{0}\right), b\right)$ where $a>0, b>0, \phi\left(t_{0}\right) \in E^{n}$, and

$$
B\left(\phi\left(t_{0}\right), b\right)=\left\{x \in E^{n}: D\left(x, \phi\left(t_{0}\right)\right) \leq b\right\} .
$$

Definition 3.1. A mapping $x: T_{0} \rightarrow E^{n}$ is a solution to the problem (1) if it is levelwise continuous and satisfies the integral equation

$$
x(t)=\phi\left(t_{0}\right)+\int_{t_{0}}^{t} h(s, x(s)) d s+\int_{t_{0}}^{t} \int_{t_{0}}^{u} q(u, s, x(s)) d s d u+\int_{t_{0}}^{t} Q\left(u, t_{0}, \phi\right) d u \text {, for all } t \in T_{0} .
$$

Assume that the following conditions hold.

(A) $h: J \rightarrow E^{n}$ is levelwise continuous and for any pair $(t, x),(t, y) \in J$ and $\alpha \in[0,1]$, we have

$$
d\left([h(t, x)]^{\alpha},[h(t, y)]^{\alpha}\right) \leq k_{h} d\left([x]^{\alpha},[y]^{\alpha}\right),
$$

where $k_{h}$ is a given constant.

(B) $q: J_{0} \rightarrow E^{n}$ is levelwise continuous and for any pair $\left(t, s, x_{1}\right),\left(t, s, x_{2}\right) \in J_{0},-b \leq s \leq t \leq b$ and $\alpha \in[0,1]$, we have

$$
d\left(\left[q\left(t, s, x_{1}\right)\right]^{\alpha},\left[q\left(t, s, x_{2}\right)\right]^{\alpha}\right) \leq k_{q}\left[d\left(\left[x_{1}\right]^{\alpha},\left[x_{2}\right]^{\alpha}\right)\right],
$$

where $k_{q}$ is a given constant. 
(C) Let $K=\max \left\{k_{h}, k_{q}\right\}$ be such that $0<K<1$.

Theorem 3.1. If the conditions $(\mathrm{A})-(\mathrm{C})$ hold, then there exists a unique solution $x=x(t)$ of (1) defined on the interval $t_{0} \leq t<t_{0}+\beta$.

Proof. Let $0<L<\beta$ be given. Therefore $t_{0} \leq t \leq t_{0}+L$.

Let

$$
\delta=\min \left\{L, \sqrt{\left(\frac{M+M_{2}}{M_{1}}\right)^{2}+\frac{2 b}{M_{1}}}-\left(\frac{M+M_{2}}{M_{1}}\right)\right\},
$$

where $M=D\left(h\left(t, \phi\left(t_{0}\right)\right), \hat{0}\right), \hat{0} \in E^{n}$, such that $\hat{0}(t)=1$ for $t=0$ and 0 otherwise, and for any $(t, x) \in J$ and $M_{1}=D\left(q\left(u, s, \phi\left(t_{0}\right)\right), \hat{0}\right)$ for any $\left(u, s, \phi\left(t_{0}\right)\right) \in J_{0}$, and $M_{2}=D\left(Q\left(u, t_{0}, \phi\left(t_{0}\right)\right), \hat{0}\right)$ for any $\left(u, t_{0}, \phi\left(t_{0}\right)\right) \in J_{0}$.

We will show that the sequence of functions defined inductively on $\left[t_{0}, t_{0}+L\right]$ by

$$
\begin{aligned}
x_{0}(t) \equiv & \phi\left(t_{0}\right), \quad t \in T_{0}, \\
x_{n}(t)= & \phi\left(t_{0}\right)+\int_{t_{0}}^{t} h\left(s, x_{n-1}(s)\right) d s+\int_{t_{0}}^{t} \int_{t_{0}}^{u} q\left(u, s, x_{n-1}(s)\right) d s d u \\
& +\int_{t_{0}}^{t} Q\left(u, t_{0}, \phi\right) d u, \quad n=1,2,3, \ldots,
\end{aligned}
$$

From (2), it follows that, for $n=1$,

$$
x_{1}(t)=\phi\left(t_{0}\right)+\int_{t_{0}}^{t} h\left(s, \phi\left(t_{0}\right)\right) d s+\int_{t_{0}}^{t} \int_{t_{0}}^{u} q\left(u, s, \phi\left(t_{0}\right)\right) d s d u+\int_{t_{0}}^{t} Q\left(u, t_{0}, \phi\left(t_{0}\right)\right) d u,
$$

which proves that $x_{1}(t)$ is levelwise continuous on $\left|t-t_{0}\right| \leq L$ and hence on $\left|t-t_{0}\right| \leq \delta$. Moreover, for any $\alpha \in[0,1]$, we have

$$
\begin{aligned}
d\left(\left[x_{1}(t)\right]^{\alpha},\left[x_{0}(t)\right]^{\alpha}\right)= & d\left(\left[\phi\left(t_{0}\right)+\int_{t_{0}}^{t} h\left(s, \phi\left(t_{0}\right)\right) d s+\int_{t_{0}}^{t} \int_{t_{0}}^{u} q\left(u, s, \phi\left(t_{0}\right)\right) d s d u\right.\right. \\
& \left.\left.+\int_{t_{0}}^{t} Q\left(u, t_{0}, \phi\left(t_{0}\right)\right) d u\right]^{\alpha},\left[\phi\left(t_{0}\right)\right]^{\alpha}\right) \\
\leq & \int_{t_{0}}^{t} d\left(\left[h\left(s, \phi\left(t_{0}\right)\right)\right]^{\alpha}, \hat{0}\right) d s+\int_{t_{0}}^{t} \int_{t_{0}}^{u} d\left(\left[q\left(u, s, \phi\left(t_{0}\right)\right)\right]^{\alpha}, \hat{0}\right) d s d u \\
& +\int_{t_{0}}^{t} d\left(\left[Q\left(u, t_{0}, \phi\left(t_{0}\right)\right)\right]^{\alpha}, \hat{0}\right) d u
\end{aligned}
$$


and by the definition of $D$, we get

$$
\begin{aligned}
D\left(x_{1}(t), x_{0}(t)\right) \leq & \int_{t_{0}}^{t} D\left(h\left(s, \phi\left(t_{0}\right)\right), \hat{0}\right) d s+\int_{t_{0}}^{t} \int_{t_{0}}^{u} D\left(q\left(u, s, \phi\left(t_{0}\right)\right), \hat{0}\right) d s d u \\
& +\int_{t_{0}}^{t} D\left(Q\left(u, t_{0}, \phi\left(t_{0}\right)\right), \hat{0}\right) d u \\
\leq & \left(M+M_{2}\right)\left|t-t_{0}\right|+M_{1} \frac{\left|t-t_{0}\right|^{2}}{2 !} \\
\leq & \left(M+M_{2}\right) \delta+M_{1} \frac{\delta^{2}}{2 !} \\
\leq & b .
\end{aligned}
$$

Now, assume that $x_{n-1}(t)$ is levelwise continuous on $\left|t-t_{0}\right| \leq \delta$ and that

$$
D\left(x_{n-1}(t), x_{0}(t)\right) \leq b .
$$

From (2), we deduce that $x_{n}(t)$ is levelwise continuous on $\left|t-t_{0}\right| \leq \delta$ and that

$$
D\left(x_{n}(t), x_{0}(t)\right) \leq b .
$$

Consequently, we conclude that $x_{n}(t)$ consists of levelwise continuous mappings on $\mid t-$ $t_{0} \mid \leq \delta$ and that

$$
\left(t, x_{n}(t)\right) \in J \text { and }\left(t, s, x_{n}(t)\right) \in J_{0}, \quad\left|t-t_{0}\right| \leq \delta, n=1,2, \ldots,
$$

Let us prove that there exists a fuzzy set-valued mapping $x:\left[t_{0}, t_{0}+L\right] \rightarrow E^{n}$ such that $D\left(x_{n}(t), x(t)\right) \rightarrow$ 0 uniformly on $\left|t-t_{0}\right| \leq \delta$ as $n \rightarrow \infty$. For $n=2$, from (2),

$$
x_{2}(t)=\phi\left(t_{0}\right)+\int_{t_{0}}^{t} h\left(s, x_{1}(s)\right) d s+\int_{t_{0}}^{t} \int_{t_{0}}^{u} q\left(u, s, x_{1}(s)\right) d s d u+\int_{t_{0}}^{t} Q\left(u, t_{0}, \phi\left(t_{0}\right)\right) d u .
$$

From (3) and (5), we have

$$
\begin{aligned}
d\left(\left[x_{2}(t)\right]^{\alpha},\left[x_{1}(t)\right]^{\alpha}\right) & \\
= & d\left(\left[\int_{t_{0}}^{t} h\left(s, x_{1}(s)\right) d s+\int_{t_{0}}^{t} \int_{t_{0}}^{u} q\left(u, s, x_{1}(s)\right) d s d u+\int_{t_{0}}^{t} Q\left(u, t_{0}, \phi\left(t_{0}\right)\right) d u\right]^{\alpha},\right. \\
& \left.\quad\left[\int_{t_{0}}^{t} h\left(s, \phi\left(t_{0}\right)\right) d s+\int_{t_{0}}^{t} \int_{t_{0}}^{u} q\left(u, s, \phi\left(t_{0}\right)\right) d s d u+\int_{t_{0}}^{t} Q\left(u, t_{0}, \phi\left(t_{0}\right)\right) d u\right]^{\alpha}\right) \\
\leq & k_{h} \int_{t_{0}}^{t} d\left(\left[x_{1}(s)\right]^{\alpha},\left[\phi\left(t_{0}\right)\right]^{\alpha}\right) d s+k_{q} \int_{t_{0}}^{t} \int_{t_{0}}^{u} d\left(\left[x_{1}(s)\right]^{\alpha},\left[\phi\left(t_{0}\right)\right]^{\alpha}\right) d s d u,
\end{aligned}
$$

So by the definition of $D$, we have

$$
D\left(x_{2}(t), x_{1}(t)\right) \leq k_{h} \int_{t_{0}}^{t} D\left(x_{1}(s), \phi\left(t_{0}\right)\right) d s+k_{q} \int_{t_{0}}^{t} \int_{t_{0}}^{u} D\left(x_{1}(s), \phi\left(t_{0}\right)\right) d s d u .
$$


Now, we can apply the first inequality (4) in the right-hand side of (6) to get

$$
\begin{aligned}
& D\left(x_{2}(t), x_{1}(t)\right) \\
& \quad \leq\left(M+M_{2}\right) K \frac{\left|t-t_{0}\right|^{2}}{2 !}+M_{1} K \frac{\left|t-t_{0}\right|^{3}}{3 !}+\left(M+M_{2}\right) K \frac{\left|t-t_{0}\right|^{3}}{3 !}+M_{1} K \frac{\left|t-t_{0}\right|^{4}}{4 !} \\
& \quad \leq K\left[\left(M+M_{2}\right) \frac{\delta^{2}}{2 !}+\left(M+M_{1}+M_{2}\right) \frac{\delta^{3}}{3 !}+M_{1} \frac{\delta^{4}}{4 !}\right] .
\end{aligned}
$$

Starting from (4) and (7), assume that

$$
\begin{aligned}
& D\left(x_{n}(t), x_{n-1}(t)\right) \\
& \leq K^{n-1}\left[{ }^{(n-1)} C_{0}\left(M+M_{2}\right) \frac{\delta^{n}}{n !}+\left[{ }^{(n-1)} C_{1}\left(M+M_{2}\right)+{ }^{(n-1)} C_{0} M_{1}\right] \frac{\delta^{n+1}}{(n+1) !}+\cdots\right. \\
&\left.+\left[{ }^{(n-1)} C_{n-1}\left(M+M_{2}\right)+{ }^{(n-1)} C_{n-2} M_{1}\right] \frac{\delta^{2 n-1}}{(2 n-1) !}+M_{1} \frac{\delta^{2 n}}{2 n !}\right]
\end{aligned}
$$

and we prove that such an inequality holds for $D\left(x_{n+1}(t), x_{n}(t)\right)$. Indeed, from (2) and the assumptions, it follows that

$$
\begin{aligned}
& d\left(\left[x_{n+1}(t)\right]^{\alpha},\left[x_{n}(t)\right]^{\alpha}\right)= \\
& \leq k_{h} \int_{t_{0}}^{t} d\left(\left[x_{n}(s)\right]^{\alpha},\left[x_{n-1}(s)\right]^{\alpha}\right) d s+k_{q} \int_{t_{0}}^{t} \int_{t_{0}}^{u} d\left(\left[x_{n}(s)\right]^{\alpha},\left[x_{n-1}(s)\right]^{\alpha}\right) d s d u,
\end{aligned}
$$

for any $\alpha \in[0,1]$ and from the condition on $D$, we have

$$
D\left(x_{n+1}(t), x_{n}(t)\right) \leq k_{h} \int_{t_{0}}^{t} D\left(x_{n}(s), x_{n-1}(s)\right) d s+k_{q} \int_{t_{0}}^{t} \int_{t_{0}}^{u} D\left(x_{n}(s), x_{n-1}(s)\right) d s d u .
$$

According to (8), we get

$$
\begin{aligned}
D\left(x_{n+1}(t), x_{n}(t)\right) & \\
\leq & K^{n}\left[{ }^{n} C_{0}\left(M+M_{2}\right) \frac{\delta^{n+1}}{(n+1) !}+\left[{ }^{n} C_{1}\left(M+M_{2}\right)+{ }^{n} C_{0} M_{1}\right] \frac{\delta^{n+2}}{(n+2) !}\right. \\
& \left.+\cdots+\left[{ }^{n} C_{n}\left(M+M_{2}\right)+{ }^{n} C_{n-1} M_{1}\right] \frac{\delta^{2 n+1}}{(2 n+1) !}+M_{1} \frac{\delta^{2 n+2}}{(2 n+2) !}\right] .
\end{aligned}
$$

Consequently, inequality (8) holds for $n=1,2, \cdots$ We can also write

$$
\begin{aligned}
& D\left(x_{n}(t), x_{n-1}(t)\right) \\
& \leq \frac{K^{n}}{K}\left[{ }^{n-1} C_{0}\left(M+M_{2}\right) \frac{\delta^{n}}{n !}\left[{ }^{(n-1)} C_{1}\left(M+M_{2}\right)+{ }^{(n-1)} C_{0} M_{1}\right] \frac{\delta^{n+1}}{(n+1) !}+\cdots\right. \\
& \left.\quad+\left[{ }^{(n-1)} C_{(n-1)}\left(M+M_{2}\right)+{ }^{(n-1)} C_{n-2} M_{1}\right] \frac{\delta^{2 n-1}}{(2 n-1) !}+M_{1} \frac{\delta^{2 n}}{(2 n) !}\right],
\end{aligned}
$$

for $n=1,2, \ldots$, and $\left|t-t_{0}\right| \leq \delta$. 
Let us mention that

$$
x_{n}(t)=x_{0}(t)+\left[x_{1}(t)-x_{0}(t)\right]+\cdots+\left[x_{n}(t)-x_{n-1}(t)\right]
$$

which implies that the sequence $\left\{x_{n}(t)\right\}$ and the series

$$
x_{0}(t)+\sum_{n=1}^{\infty}\left[x_{n}(t)-x_{n-1}(t)\right]
$$

have the same convergence properties. From (9), it follows that $D\left(x_{n}(t), x_{n-1}(t)\right) \rightarrow 0$ uniformly on $\left|t-t_{0}\right| \leq \delta$ as $n \rightarrow \infty$. Hence, there exists a fuzzy set-valued mapping $x:\left[t_{0}, t_{0}+L\right] \rightarrow$ $E^{n}$ such that $D\left(x_{n}(t), x(t)\right) \rightarrow 0$ uniformly on $\left|t-t_{0}\right| \leq \delta$ as $n \rightarrow \infty$. From the assumptions, we get

$$
d\left(\left[h\left(t, x_{n}(t)\right)\right]^{\alpha},[h(t, x(t))]^{\alpha}\right) \leq k_{h} d\left(\left[x_{n}(t)\right]^{\alpha},[x(t)]^{\alpha}\right)
$$

for any $\alpha \in[0,1]$, and so

$$
D\left(h\left(t, x_{n}(t)\right), h(t, x(t))\right) \leq k_{h} D\left(x_{n}(t), x(t)\right) \rightarrow 0
$$

uniformly on $\left|t-t_{0}\right| \leq \delta$ as $n \rightarrow \infty$. Furthermore,

$$
d\left(\left[q\left(t, s, x_{n}(s)\right)\right]^{\alpha},[q(t, s, x(s))]^{\alpha}\right), \leq k_{q} d\left(\left[x_{n}(s)\right]^{\alpha},[x(s)]^{\alpha}\right)
$$

for any $\alpha \in[0,1]$, and

$$
D\left(q\left(t, s, x_{n}(s)\right), q(t, s, x(s))\right) \leq k_{q} D\left(x_{n}(s), x(s)\right) \rightarrow 0
$$

uniformly on $\left|t-t_{0}\right| \leq \delta$ as $n \rightarrow \infty$.

Taking (10) and (11) into account, from (2), we obtain,

$$
x(t)=\phi\left(t_{0}\right)+\int_{t_{0}}^{t} h(s, x(s)) d s+\int_{t_{0}}^{t} \int_{t_{0}}^{u} q(u, s, x(s)) d s d u+\int_{t_{0}}^{t} Q\left(u, t_{0}, \phi\left(t_{0}\right)\right) d u
$$

for $n \rightarrow \infty$,

Consequently, there is at least one levelwise continuous solution of (1).

We want to prove now that this solution is unique, that is, from

$$
y(t)=\phi\left(t_{0}\right)+\int_{t_{0}}^{t} h(s, y(s)) d s+\int_{t_{0}}^{t} \int_{t_{0}}^{u} q(u, s, y(s)) d s d u+\int_{t_{0}}^{t} Q\left(u, t_{0}, \phi\left(t_{0}\right)\right) d u
$$

on $\left|t-t_{0}\right| \leq \delta$, we want to show that $D(x(t), y(t)) \equiv 0$. Indeed, from (2) and (12), we have

$$
d\left([y(t)]^{\alpha},\left[x_{n}(t)\right]^{\alpha}\right) \leq k_{h} \int_{t_{0}}^{t} d\left([y(s)]^{\alpha},\left[x_{n-1}(s)\right]^{\alpha}\right) d s+k_{q} \int_{t_{0}}^{t} \int_{t_{0}}^{u} d\left([y(s)]^{\alpha},\left[x_{n-1}(s)\right]^{\alpha}\right) d s d u
$$

for any $\alpha \in[0,1], n=1,2, \ldots$ 
By the definition of $D$, we have

$$
D\left(y(t), x_{n}(t)\right) \leq K \int_{t_{0}}^{t} D\left(y(s), x_{n-1}(s)\right) d s+K \int_{t_{0}}^{t} \int_{t_{0}}^{u} D\left(y(s), x_{n-1}(s)\right) d s d u
$$

But $D\left(y(t), x_{0}(t)\right) \leq b$ on $\left|t-t_{0}\right| \leq \delta, y(t)$ being a solution of (12). It follows from (13) that

$$
\begin{aligned}
D\left(y(t), x_{1}(t)\right) & \leq K \int_{t_{0}}^{t} D\left(y(s), x_{0}(s)\right) d s+K \int_{t_{0}}^{t} \int_{t_{0}}^{u} D\left(y(s), x_{0}(s)\right) d s d u \\
& \leq K b\left[\left|t-t_{0}\right|+\frac{\left|t-t_{0}\right|^{2}}{2 !}\right]
\end{aligned}
$$

on $\left|t-t_{0}\right| \leq \delta$. Also,

$$
\begin{aligned}
D\left(y(t), x_{2}(t)\right) & \leq K \int_{t_{0}}^{t} D\left(y(s), x_{1}(s)\right) d s+K \int_{t_{0}}^{t} \int_{t_{0}}^{u} D\left(y(s), x_{1}(s)\right) d s d u \\
& \leq K^{2} b\left[\frac{\left|t-t_{0}\right|^{2}}{2 !}+2 \frac{\left|t-t_{0}\right|^{3}}{3 !}+\frac{\left|t-t_{0}\right|^{4}}{4 !}\right]
\end{aligned}
$$

on $\left|t-t_{0}\right| \leq \delta$. Now assume that

$$
\begin{aligned}
D\left(y(t), x_{n}(t)\right) \leq & K^{n} b\left[{ }^{(n-1)} C_{0} \frac{\left|t-t_{0}\right|^{n}}{n !}+{ }^{(n-1)} C_{1} \frac{\left|t-t_{0}\right|^{n+1}}{(n+1) !}\right. \\
& \left.+\cdots+{ }^{(n-1)} C_{n} \frac{\left|t-t_{0}\right|^{2 n}}{(2 n) !}\right]
\end{aligned}
$$

on the interval $\left|t-t_{0}\right| \leq \delta$. From

$$
D\left(y(t), x_{n+1}(t)\right) \leq K \int_{t_{0}}^{t} D\left(y(s), x_{n}(s)\right) d s+K \int_{t_{0}}^{t} \int_{t_{0}}^{u} D\left(y(s), x_{n}(s)\right) d s d u
$$

and (14), one obtains

$$
D\left(y(t), x_{n+1}(t)\right) \leq K^{n+1} b\left[{ }^{n} C_{0} \frac{\left|t-t_{0}\right|^{n+1}}{(n+1) !}+{ }^{n} C_{1} \frac{\left|t-t_{0}\right|^{n+2}}{(n+2) !}+\cdots+{ }^{n} C_{n} \frac{\left|t-t_{0}\right|^{2 n+1}}{(2 n+1) !}\right]
$$

Consequently, (14) holds for any $n$, which leads to the conclusion

$$
D\left(y(t), x_{n}(t)\right)=D\left(x(t), x_{n}(t)\right) \rightarrow 0
$$

on the interval $\left|t-t_{0}\right| \leq \delta$ as $n \rightarrow \infty$. Thus, there exists an unique solution on $\left[t_{0}, t_{0}+L\right]$. Since $\mathrm{L}$ is arbitrary in $(0, \beta)$. Therefore, there exists an unique solution on $\left[t_{0}, t_{0}+\beta\right)$.

Example. Consider the fuzzy Volterra integral equation with infinite delay

$$
\begin{aligned}
x^{\prime}(t) & =\left(\frac{5}{6}-\frac{6}{11 e}\right) x(t)+\int_{-\infty}^{t} e^{(s-t-1)} x(s) d s, \\
{[x(t)]^{\alpha} } & =[\phi(t)]^{\alpha}=e^{2 t}[(1+\alpha),(3-\alpha)], \quad t \in(-\infty, 0], \alpha \in[0,1] .
\end{aligned}
$$


Since the conditions (A)-(C) hold, from Theorem 3.1 the above equation has a unique solution.

\section{Acknowledgement}

The work is supported by Department of Science and Technology, New Delhi, India.

\section{References}

[1] R. P. Agarwal, D. O. Regan and V. Laksmikantham, Fuzzy Volterra integral equations: a Stacking theorem approach, Applicable Analysis 83 521-532 (2004).

[2] R. J. Aumann, Integrals of set-valued functions, Journal of Mathematical Analysis and Applications 12 (1965), 1-12.

[3] K. Balachandran and P. Prakash, Existence of solutions of nonlinear fuzzy volterra-Fredholm integral equations, Indian Journal of Pure Applied Mathematical 33 (2002), 329-343.

[4] P. Diamond, Theory and applications of fuzzy Volterra integral equations, IEEE Transactions on Fuzzy Systems 10 (2002), 97-101.

[5] O. Kaleva, Fuzzy differential equations, Fuzzy Sets and Systems 24 (1987), 301-317.

[6] O. Kaleva, The Cauchy problem for fuzzy differential equations, Fuzzy Sets and Systems 35 (1990), 389-396.

[7] A. Kandel and W. J. Byatt, Fuzzy differential equations, Proceedings International Conference Cybernetics and Society, Tokyo, (1978), 1213-1216.

[8] C. V. Negoita and D. A. Ralescu, Applications of Fuzzy Sets to Systems Analysis, Wiley, New York, Toronto, 1975.

[9] M. L. Puri and D. A. Ralescu, Fuzzy random variables, Journal of Mathematical Analysis and Applications 114 (1986), 409-422.

[10] Shiji Song, Qin-Yu Liu, and Qi-chun Xu, Existence and comparison theorems to Volterra fuzzy integral equation in $\left(E^{n}, D\right)$, Fuzzy Sets and Systems 104 (1999), 315-321.

[11] X. Xiaoping and F. Yongqiang, On the structure of solutions for fuzzy initial value problem, Fuzzy Sets and Systems 157 (2006), 212-229.

Department of Mathematics, Periyar University, Salem - 636 011, India.

E-mail:kp-prakash@lycos.com

Department of Mathematics, Periyar University, Salem - 636 011, India.

E-mail: 